\title{
EL GOBIERNO CORPORATIVO COMO FACTOR CRÍTICO DEL ÉXITO EMPRESARIAL EN EL SECTOR SALUD
}

David Jervis Jálabe Universidad de la Costa Colombia

Julio Orozco Africano Universidad de la Costa Colombia

Luis Ortiz Ibañez Universidad de la Costa Colombia

Jannina Alvis Zakzuk ALZAK Foundation Colombia

Tulia Beltran Venegas Universidad de la Costa Colombia 
Panorama Económico, Vol. 27 - No. 3 (Julio - Septiembre de 2019), pp. 734-757

David Jervis Jálabe

Julio Orozco Africano

Luis Ortiz Ibañez

Jannina Alvis Zakzuk

Tulia Beltran Venegas

\title{
El gobierno corporativo como factor crítico del éxito empresarial en el sector salud
}

\section{Resumen}

Los beneficios que el Buen Gobierno Corporativo (BGC) acarrea para las empresas, como las características propias de su estructuración y su fortalecimiento que mayor consenso han logrado en empresarios y estudiosos, permitió determinar su presencia en dos empresas del aseguramiento en salud de la región caribe colombiana, además de valorar la relación de causa-efecto entre ambas desde la percepción de sus respectivos Gerentes Generales. Se realizaron de entrevistas estructuradas, a partir de las cuales se buscó probar la hipótesis que el éxito empresarial exhibido por las dos empresas, se encuentra fuertemente relacionado con la estructuración y fortalecimiento de un BGC. Para el caso de las empresas analizadas, los resultados muestran que aunque no se encontraron la totalidad de las características recomendadas en la literatura, ni la percepción de relación entre ellas y todos los logros organizacionales referidos, si se encontró evidencia de la relación entre las características presentes y los desarrollos esperados que constituyen la base de su éxito organizacional.

Palabras clave: Gobierno corporativo, mejores prácticas de gobierno corporativo, grupos de interés, accionistas, conducta corporativa, éxito empresarial.

\section{La gouvernance d'entreprise comme facteur critique du succès des entreprises dans le secteur de la santé}

\section{Résumé:}

Les avantages que la bonne gouvernance d'entreprise (BGC) entraîne pour les entreprises, tels que les caractéristiques de sa structuration et son renforcement qu'un plus grand consensus a été atteint chez les hommes d'affaires et les universitaires, ont permis de déterminer sa présence dans deux compagnies d'assurance maladie dans la région colombienne des Caraïbes, en plus d'évaluer la relation de cause à effet entre les deux à partir de la perception de leurs directeurs généraux respectifs. Des entretiens structurés ont été menés, à partir desquels on a cherché à tester l'hypothèse selon laquelle la réussite commerciale des deux sociétés est fortement liée à la structuration et au renforcement d'un BGC.Dans le cas des entreprises analysées, les résultats montrent que, bien que toutes les caractéristiques recommandées dans la littérature n'aient pas été trouvées, ni la perception de la relation entre elles et toutes les réalisations organisationnelles mentionnées, si des preuves de la relation entre les caractéristiques ont été trouvées présente et les développements attendus qui sont à la base de son succès organisationnel.

Mots clés: Gouvernance d'entreprise, meilleures pratiques de gouvernance d'entreprise, groupes d'intérêt, actionnaires, conduite des entreprises, réussite commerciale.

\section{Corporate governance as a critical factor of business success in the health sector}

\begin{abstract}
The benefits that Good Corporate Governance (BGC) entails for companies, such as the characteristics of its structuring and its strengthening that greater consensus has been achieved in businessmen and scholars, allowed to determine its presence in two health insurance companies in the Caribbean region Colombian, in addition to assessing the cause-effect relationship between the two from the perception of their respective General Managers. Structured interviews were carried out, from which it was sought to test the hypothesis that the business success exhibited by the two companies is strongly related to the structuring and strengthening of a BGC. In the case of the companies analyzed, the results show that although all the characteristics recommended in the literature were not found, nor the perception of the relationship between them and all the organizational achievements referred to, if evidence of the relationship between the characteristics was found present and the expected developments that form the basis of its organizational success.
\end{abstract}

Keywords: Corporate governance, best corporate governance practices, interest groups, shareholders, corporate conduct, business success. 


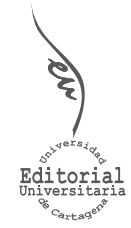

\section{Artículo de Investigación}

\section{El gobierno corporativo como factor crítico del éxito empresarial en el sector salud}

INFORMACIÓN DEL ARTÍCULO

Recepción del artículo: 22/12/2018

Concepto de evaluación: 23/03/2019

Aceptación del artículo: 30/04/2019
David Jervis Jálabe Universidad de la Costa, Colombia

Julio Orozco Africano AUniversidad de la Costa, Colombia

Luis Ortiz lbañez Universidad de la Costa, Colombia

Jannina Alvis Zakzuk ALZAK Foundation, Colombia

Tulia Beltran Venegas Universidad de la Costa, Colombia

\section{INTRODUCCIÓN}

En el presente artículo se busca determinar el grado en que las dos empresas escogidas para el estudio de casos, deben su éxito empresarial al desarrollo e implementación de buenas prácticas de gobierno corporativo, según parámetros y criterios recopilados de estudios académicos y de mercado, así como las recomendaciones de instituciones referentes en el tema. La mejora de las prácticas de gobierno corporativo en organizaciones privadas y estatales es un fenómeno internacional que continúa presente en la agenda de inversionistas, empresarios, reguladores y académicos a nivel global enfocados en incrementar la creación de valor a las empresas y su impacto en la sociedad (Shleifer \& Vishny, 1989). Las razones que impulsan la búsqueda de mejoras en el gobierno corporativo son tan numerosas y variadas como sus métodos de implementación. En muchos casos, la adopción de medidas específicas en la materia, ocurre tras un período relativamente largo destinado a lograr una mayor comprensión de los costos y los beneficios involucrados. Durante este período, los responsables clave de la empresa van apreciando cada vez más las ventajas que puede ofrecer la mejora del gobierno corporativo tanto al negocio, como a los diversos interesados, a nivel interno y externo (Wong, 2008). Las organizaciones con un buen gobierno corporativo tienen la capacidad de mantener servicios de alta calidad y de mejorar continuamente (Carney, 2005). Un gobierno corporativo pobre ha contribuido a menudo a serios fracasos financieros y en 
la operación de la empresa. El creciente debate sobre responsabilidad social corporativa y la muerte empresarial lleva este asunto a una perspectiva cada vez más sombría si no se toman las medidas correctivas necesarias (Morck, Wolfenzon, \& Yeung, 2004).

El problema planteado se aborda desde tres (3) dimensiones: 1. Resultados de estudios académicos y de mercado, sobre los beneficios del gobierno corporativo; 2 . Recopilación exhaustiva de los criterios de buenas prácticas de instituciones referentes y 3 . Estudio de los casos de dos empresas promotoras de salud del régimen subsidiado. El gobierno corporativo es el conjunto de reglas que regula las relaciones establecidas entre las diferentes partes interesadas de la empresa con el fin de garantizar que cada una de ellas reciba una parte equitativa de sus dividendos, sean estos económicos, sociales o ambientales (Aguirre Brand, 2010). Lo anterior resulta fundamental para generar los incentivos adecuados que atraigan los inversionistas cuyo capital es necesario para el desarrollo de la empresa, lo cual no ocurre en forma automática debido a la existencia de asimetrías en la información y la imposibilidad de implementar contratos perfectos o cuasi-perfectos frente a cada una de las posibles eventualidades futuras y riesgos de fallos. Son muchos los aspectos que conforman el gobierno corporativo y condicionan, de una u otra forma, el desempeño y el valor de la empresa. Además, un adecuado gobierno corporativo favorece el desarrollo del sistema financiero y el crecimiento económico, al permitir el encuentro, dentro de un marco de confianza, de capitales de bajo costo y una administración cuya gestión eficaz de genera rendimientos razonables y maximiza el valor de la inversión, con mínimos riesgos operativos (Williamson, 1988).

En el sector salud en Colombia, se agregan otras situaciones que se asemejan al acceso a capitales de bajo costo, en forma de costos razonables de operación a pesar de altos costos transaccionales, que tienen en la confianza en el buen gobierno corporativo su más eficaz herramienta.

Entre las principales recomendaciones para el desarrollo y fortalecimiento del BGC halladas en la literatura revisada encontramos: la distribución equitativa de las rentas de capital, la asignación eficiente de recursos, la retención de los derechos de propiedad por parte de los accionistas, la alineación de los intereses de los accionistas con los de la empresa, la protección de los accionistas contra la expropiación y los costos de agencia, la igualdad de derechos entre los accionistas, la garantía de acceso por parte de los accionistas y otros stakeholders a información oportuna, suficiente y adecuada respecto a las decisiones de los ejecutivos, la debida reglamentación de la adquisición del control corporativo. Una conclusión importante es que no existen modelos sencillos ni definitivos que funcionen en todos los casos y circunstancias por lo que el BGC será siempre el producto del esfuerzo denodado de los directivos y administradores por asegurar el cumplimiento de los objetivos estratégicos de la empresa sin desmedro de ninguna de sus partes interesadas.

Los estudios de casos demostraron que el BGC resultó un catalizador importante del desarrollo y crecimiento empresarial y factor contribuyente importante para el alcance de importantes beneficios corporativos, entre ellos: incremento en el valor de la empresa, captación y retención de inversionistas y diversificación del capital de trabajo, capacidad de la empresa para enfrentar y responder a las presiones del mercado, equilibrar el interés de los accionistas, resolver problemas generados por intereses propios de los asociados, los órganos de dirección y la alta gerencia, el logro de mejores resultados operativos, mejora en la reputación de la empresa, mejora del clima organizacional y disminución de la rotación del personal. 


\section{REVISIÓN DE LITERATURA}

Han pasado más de 50 años desde que Milton Friedman (1962) promulgó por primera vez lo que ahora se conoce como la "doctrina Friedman", en su libro Capitalismo y Libertad. En este sentido, la responsabilidad social es un "concepto fundamentalmente subversivo" en una sociedad libre y en cuyo marco "hay una y sólo una responsabilidad social de las empresas: utilizar sus recursos y adelantar sus actividades con el fin de incrementar sus beneficios, respetando siempre las reglas del juego, es decir, dentro de una competencia abierta y libre, sin engaño o fraude".

La doctrina Friedman llegó a ser tan poderosamente difundida y aceptada, que no sólo se utilizó para justificar las conductas corporativas maquiavélicas y las políticas gubernamentales liberales hacia la empresa en todo el mundo, sino que además sustentó una educación de negocios que moldeó generaciones enteras de líderes empresariales. Lo anterior refleja la capacidad de las ideas para influir en el comportamiento humano, hasta el punto en que muchas personas creyeron (y algunos todavía lo creen) que la doctrina Friedman era una ley de la naturaleza de la que, nos guste o no, somos incapaces de escapar.

En su obra, Friedman arremete en contra de la afirmación que ataca los negocios modernos: la separación de la propiedad y el control, a la que se acusa de causar que la empresa se haya convertido en una institución social en la que sus ejecutivos, sin el control de los accionistas, de manera irresponsable, sirven a sus propios intereses y por lo tanto no generan bienestar social. Según Friedman, esta premisa es falsa, ya que las empresas son sólo el instrumento de unos individuos, sus accionistas, y los negocios como un todo carecen de "responsabilidades", obligación que únicamente pueden asumir los seres humanos. En cambio, al pretender que las empresas tengan fines sociales se las está empujando a un verdadero divorcio entre propiedad y control, al buscarse fines diferentes a la rentabilidad económica de los accionistas, lo que erosiona la naturaleza básica y el carácter de la sociedad capitalista. Tal pensamiento, según Friedman, nos aleja de la sociedad individualista en dirección al estado corporativo.

Medio siglo después, estamos empezando a entender no sólo que la apreciación de Friedman está errada, sino también en qué consiste su error: las empresas y los negocios que ellas desarrollan no sólo tienen responsabilidades distintas a las nuestras como propietarios, directivos o empleados, sino que además la sociedad se nutre fundamentalmente de dichas responsabilidades. Como bien lo explica Colin Mayer (2013) en su libro Compromiso Empresarial, la empresa es una entidad de notables características. Puede contratar y ser contratada, emplear y ser empleada, demandar y ser demandada - al igual que el resto de nosotros. De hecho, puede hacer muchas más cosas de las que nosotros somos capaces, como proporcionar grados de compromiso a los que nosotros, como individuos, sólo podemos aspirar, y en ese proceso, superar deficiencias y fracasos que de otra manera nos empobrecerían. Y todo esto lo puede hacer debido precisamente a la separación entre propiedad y control. Lo que en un contexto convencional, y a los ojos de Friedman, es considerado como una deficiencia de la empresa, en realidad es un atributo que le permite equilibrar el grado de compromiso que ofrece a las diferentes partes con el control que ejerce sobre ellas.

Siguiendo estas ideas, Michael Klein (2013), Doctor en Economía de la Universidad de Bonn (Alemania) y profesor de la Escuela de Finanzas y Administración de Frankfurt (Alemania), ha descrito los dilemas que enfrentan aquellos que ejecutan y regulan la 
empresa moderna. Conflictos que hacen que las recetas convencionales para la empresa promedio resulten defectuosas en su esencia. Partiendo del hecho de que las empresas modernas son estructuras complejas que para ser eficientes requieren que los intereses de sus partes interesadas (stakeholders) se alineen convenientemente, el Doctor Klein nos hace conscientes de que dicha complejidad se aumenta al incluir entre las partes interesadas tradicionales (clientes, empleados, proveedores, accionistas -stakeholders y acreedores) a las más recientemente reconocidas: comunidad y medio ambiente. Esta compleja alineación de intereses debería ser llevada a cabo por la administración en el proceso de manejar la empresa, echando mano de su discrecionalidad, pero en el marco de reglas derivadas de la plataforma estratégica corporativa (misión, visión, valores, principios, objetivos, políticas, directivas, reglamentos y procesos y procedimientos). Estas reglas, así como los procesos y arreglos organizacionales que vienen con ellas son el dominio del gobierno corporativo.

Según Klein, la necesidad del gobierno corporativo se hace más patente cuanto más complejo es el contrato que garantiza a sus financiadores el retorno de la inversión. Cuando el capital de la empresa proviene, por ejemplo, de una deuda bancaria o comercial, el contrato es relativamente simple. Tanto el capital como los intereses pactados deben ser pagados al acreedor siguiendo un cronograma fijo de cuotas pre-establecidas y la empresa debe aportar algún tipo de garantía del cumplimiento de dichos pagos en forma de activos o pólizas que aseguran el capital prestado. Si el dinero no se paga según lo contratado, el prestatario entra en mora y el acreedor puede hacer efectivas las garantías o incluso tomar posesión de la empresa y su capacidad productiva. En cambio, cuando la financiación proviene de capital aportado por socios, asociados o accionistas, el contrato es bastante más complejo. Sus retornos no son "fijos" (como las cuotas de un préstamo) y así como pueden ser mayores a lo proyectado (ventaja de la que carecen los prestamistas), también pueden serlo muy por debajo de lo esperado. A lo anterior se le agrega el riesgo que la empresa oculte los beneficios o que los desvíen en orden de no entregar los dividendos debidos. En la publicación referida, Klein nos regala una cita del banquero alemán Carl Fuerstenberg (1850-1933), tomada a su vez del artículo Sobre la economía y la política de las finanzas y el control coporativo (Hellwig, 1998), en la que se caracteriza de manera inmejorable este dilema: "Los accionistas son estúpidos e impertinentes. Estúpidos porque confían su dinero a personas a las que no controlan adecuadamente. Impertinentes porque piden dividendos, y así incluso quieren ser recompensados por su estupidez"1.

Obviamente, este problema no lo enfrentan las compañías pequeñas, de un único dueño o familiares, cuyos problemas de gobernanza son de otra naturaleza, como las disputas personales y la lucha por la sucesión en el control (Quintana, 2012). Más bien son propias de las grandes empresas con muchos accionistas, donde o bien hay unos cuantos accionistas mayoritarios que ejercen el control de voto e imponen su criterio sobre los minoritarios (que casi siempre son muchos más) o, peor aún, donde la gran mayoría de los accionistas son pequeños y dispersos, quedando a merced de los administradores quienes privilegian sus propios intereses o sencillamente fracasan en maximizar el valor de la empresa (Mayer, 2013).

1.El texto originalmente citado por Hellwig es el que sigue: "Shareholders are stupid and impertinent. Stupid, because they entrust their money to people they do not adequately control. Impertinent, because they ask for dividends and thus even want to be rewarded for their stupidity." 
En cualquiera de los dos casos, el problema que emerge es el mismo: la dificultad para encontrar nuevas fuentes de financiación o bien obtenerla a un costo que permita el crecimiento adecuado de la empresa. Para atraer inversionistas, las empresas necesitan generar confianza en que los mismos serán razonablemente recompensados con tasas de retorno y valorización de la inversión por encima de las que ofrecen los bancos en sus depósitos a término fijo. Así, el estímulo clave para que las empresas fortalezcan su gobierno corporativo lo constituye la búsqueda de mayores fuentes de capital a más bajo costo, que si bien por sí solo no es suficiente para garantizar el éxito empresarial, si es un aspecto crítico (Rodríguez, 2004).

En este sentido, existen en Colombia, y especialmente en el sector de la salud, algunas circunstancias que entran a jugar como factores que aumentan la complejidad de la dinámica empresarial, tanto del lado de los aseguradores (Empresas Promotoras de Salud -EPS) como del de los proveedores (Instituciones Prestadoras de Servicios de Salud -IPS). De acuerdo al modelo de aseguramiento en salud desarrollado por el estado colombiano para prestar el servicio público de salud, en concordancia con el mandato constitucional de 1991, dicho servicio es financiado en su totalidad por el Estado, que a la vez nutre sus arcas principalmente de las contribuciones realizadas por los ciudadanos con ingresos regulares superiores a dos salarios mínimos legales vigentes y las empresas para los cuales dichas personas trabajan, en el caso de los trabajadores dependientes, en forma de un impuesto sobre la nómina y de un porcentaje de los ingresos corrientes de la nación a través del sistema general de participaciones (SGP). Dichos dineros son luego entregados a valores fijos per cápita, proporcionales al costo generado por los diversos grupos etarios, a las EPS, quienes se encargan de realizar la "compra inteligente" de servicios de salud a las IPS y de realizar los procesos de auditoría de calidad de la atención en salud y costo de la misma.

Así las cosas, y debido a que el flujo de dinero sufre de continuas e importantes irregularidades, las EPS fundamentan en su capacidad de negociación y de generación de confianza en la red de proveedores de servicios, la consecución de tarifas lo suficientemente bajas para que les permitan equilibrar sus ingresos por cada usuario afiliado con los egresos generados principalmente por la atención de aquellos que lo requieren con necesidad debido a la pérdida de su estado de salud o bienestar (Restrepo Zea, 2004).

Aunque no está documentado, es un hecho conocido por todos en el sector, que las IPS compensan el riesgo de los pagos a largo plazo de los servicios prestados con el aumento desproporcionado de las tarifas de los mismos, generando riesgos de liquidez y de solvencia en los aseguradores, asemejando esta situación a la financiación de la operación de las EPS con alto costo de capital. Además, no es infrecuente, que las IPS recurran a las vías judiciales para el pago de las obligaciones no cumplidas por los aseguradores, aumentando aún más el riesgo financiero al cargarle a las ya altas tarifas, valores correspondientes a intereses y honorarios legales.

En cambio, si existen publicaciones que demuestran que la confianza entre aseguradores y prestadores es vital para el equilibrio económico y financiero, no sólo de los primeros sino de todo el sistema (Gorbaneff, Torres, \& Contreras, 2004), asunto en el que el gobierno corporativo puede jugar un importante rol, incluso sin que esa sea la motivación de socios, directivos y administradores. 


\section{MÉTODO}

Para el desarrollo de la presente investigación se aborda el problema desde tres (3) perspectivas: 1. Revisión de los resultados de estudios académicos y de mercado que demuestran beneficios del BGC para establecer una relación de causalidad en los casos estudiados; 2. Recopilación de los criterios de buenas prácticas de instituciones referentes -incluidas la IFC, la OCDE y el Foro Global de Gobierno Corporativo- que soporten con su presencia la relación de causalidad que pueda surgir en la dimensión tres o que puedan ser recomendadas a las empresas en estudio para su implementación posterior y; 3. Estudiar los casos de dos empresas promotoras de salud del régimen subsidiado (EPS-S), ambas pertenecientes al tercer sector de la economía, una cooperativa y una mutual, pero con orígenes similares.

Para ello, se recopiló una amplia bibliografía, de más de cincuenta fuentes documentales, para abordar las dimensiones dos y tres del estudio, de manera que las entrevistas rindieran su máximo provecho en la búsqueda de los aspectos significativos de para la implementación y desarrollo del gobierno corporativo y su desarrollo posterior.

Así mismo, se elaboró una encuesta con base en la cual se realizaron entrevistas a los directivos más prominentes de las dos empresas, a fin de documentar y recrear, desde su percepción autorizada, la travesía que debieron emprender como líderes de sus respectivas organizaciones al implementar los cambios que fundamentaron y desarrollaron el gobierno corporativo, empezando por las motivaciones y terminando con los beneficios, tangibles e intangibles, cosechados a lo largo del mismo, ayudando al investigador a comprender la lógica del proceso de implementación de este aspecto fundamental del desarrollo organizacional y su influencia en el crecimiento de la entidad. Es de interés determinar en este punto si los desarrollos alcanzados se hicieron sobre motivaciones conscientes de alcanzar objetivos propuestos, o si estos últimos emergieron como un valor agregado insospechado y en tal caso si se han valorado en su verdadera dimensión.

\section{RESULTADOS}

\section{Beneficios del gobierno corporativo para las empresas}

Los académicos y los expertos en gobierno corporativo llevan tiempo intentando identificar y demostrar empíricamente que existe una relación entre las buenas prácticas de gobierno y el éxito empresarial. En el curso de nuestra revisión bibliográfica, encontramos varios estudios que intentaron analizar la manera en que las diferentes características del gobierno corporativo afectan la rentabilidad, las fluctuaciones del precio de las acciones, la valoración de una empresa y otros resultados económicos corporativos.

\subsubsection{Valor de la empresa en el mercado y rentabilidad}

Gompers, Ishii y Metrick (2001), luego de construir un índice de gobernanza lo aplicaron a cerca de 1.500 compañías por año durante los 90 encontrando una sorprendente relación entre el valor obtenido con la medición de este índice en una compañía dada y la rentabilidad de sus acciones, al igual que con el valor de la empresa. En ese mismo sentido, Klapper y Love (2004) demostraron que las mejores estructuras de gobierno corporativo en empresas de mercados emergentes mostraban una alta relación positiva con un mejor desempeño operativo y un mayor valor de mercado y más importante aún, las autoras 
proveyeron evidencia que la construcción de gobierno corporativo es más importante aún en países con ambientes legales débiles. Por otra parte, Lemmon y Lins (2003) encontraron en una muestra de 800 empresas pertenecientes a 8 países del este asiático que aquellas con estructuras débiles de gobierno corporativo perdieron valor durante la crisis económica de la región entre 10 y 20 puntos porcentuales por debajo de aquellas con mejores prácticas de gobernanza. Finalmente, Black y cols (2006) evidenciaron, en un elegante estudio, que el gobierno corporativo es un factor fundamental en el aumento de valor de mercado de las compañías coreanas que cotizan en bolsa y que existe una relación causal para ello.

También en Latinoamérica contamos con este tipo de estudios que demuestran que el fenómeno descrito también está presente en nuestro continente. En un trabajo adelantado por Da Silveira y Barros (2007) que estudió la influencia de la calidad del gobierno corporativo en el valor de mercado de 154 empresas que cotizan en bolsa en Brasil en 2002, dio como resultado, en todos los enfoques econométricos, que existe una evidente influencia positiva y significativa de la calidad del gobierno corporativo en el valor de mercado de las empresas estudiadas, a tal grado que un mejoramiento en la calidad del gobierno corporativo se traduciría en un aumento de capitalización de mercado de alrededor del 85\% al 100\%. Otro estudio (Leal \& Carvalhal da Silva, 2005), encontró que un aumento de un punto en el índice de gobierno corporativo utilizado generaba un aumento simultáneo en promedio de 6,8\% en el precio de mercado de empresas que cotizaban en bolsa de valores. El resultado fue consistente tanto para empresas en Brasil como en Chile.

Aunque estos estudios apenas aportan evidencia circunstancial, todos han demostrado una fuerte relación causa-efecto entre la existencia y fortalecimiento del gobierno corporativo y el valor de mercado de las empresas y la rentabilidad de sus acciones, al menos para las compañías que cotizan en bolsa. Las razones que podrían explicar este fenómeno han sido objeto de fuertes debates, la mayoría de los cuales se basa en si es verdadera la premisa de que adoptar buenas prácticas de gobierno tiene una influencia positiva sobre el desempeño de la empresa. Como parecen demostrar los estudios referenciados, las buenas prácticas de gobierno conllevan varias ventajas, entre ellas: mejores procesos de toma de decisiones en los niveles más altos de la organización, mejor y más transparente entorno de control y reducción de los costos de capital (Coffee Jr, 2002).

\subsubsection{Mejora en los resultados operativos}

Para las empresas que cotizan en bolsa, el beneficio del buen gobierno que se menciona más habitualmente, es su efecto sobre el valor de las acciones, la liquidez y la composición de la cartera de inversión. Estos beneficios constituyen entonces los principales factores de motivación para que los accionistas y directores aborden el proceso de mejora de las políticas y las prácticas de gobierno en las empresas que cotizan en la bolsa de valores (Cano Morales, Orduz Aguilar, \& Hoyos Ramírez, 2007).

Sin embargo, teniendo en cuenta que la mejora del modelo de gobierno también ejerce una influencia positiva en los procesos de toma de mejores decisiones comerciales y estratégicas, sería razonable pensar que las empresas mejor gobernadas lograrán mejores resultados operativos. Este posible beneficio es importante, ya que se puede aplicar tanto a las empresas que cotizan en bolsa como a las que no lo hacen, mientras que el incremento del valor de las acciones sólo es aplicable a las empresas que cotizantes (Flores Konja \& Rozas Flores, 2013). 
En un estudio reciente adelantado por la IFC (2010), en el que se comparaban los resultados económicos y operacionales de 14 empresas, pertenecientes al Círculo de Empresas de la Mesa Redonda Latinoamericana, escogidas por sus importantes avances en gobierno corporativo y otras 1.078 empresas que cotizaron en bolsa, durante el periodo 20052007, se compararon los resultados operativos obtenidos por los dos grupos de empresas utilizando para ello indicadores contables tradicionales (retorno de inversión -ROI, margen EBITDA, índice de pagos, ratio corriente, ratio de solvencia y ratio de deuda) e indicadores tradicionales del mercado de valores (capitalización en el mercado, ratio Precio/Ganancia -PE, ratio Precio/Valor -PBV, Volumen diario de acciones negociadas, índice de liquidez, rendimiento de dividendos). Los resultados fueron sorprendentes: en general por cada dólar invertido el 31 de diciembre de 1997, en una cartera con acciones de todos los miembros del Círculo de Empresas, 11 años después, se habrían obtenido US $\$ 15,45$, para un rendimiento acumulado del $1.445 \%$, excepcionalmente más altos que si hubiese invertido la misma suma en cualquier otra cartera - incluso tras la crisis financiera global de 2008 , del que solo habría obtenido US $\$ 3,42$ por cada dólar invertido en una cartera de todas las empresas latinoamericanas.

La explicación para la existencia de diferencias tan evidentes se ha buscado en las fortalezas de las que el gobierno corporativo dota a las organizaciones, entre ellas (Turrent \& del Carmen, 2012):

- Un proceso de institucionalización, que permite que la conducción de la empresa no dependa tanto de personas específicas, generando patrones de decisión más estables, predecibles y, por tanto, confiables.

- Mayor confianza de la comunidad de inversores

- Mejor acceso a condiciones de crédito más convenientes, lo que incluye el financiamiento a largo plazo de los bancos de desarrollo

- Mayor reconocimiento de las partes interesadas (stakeholders), incluyendo instituciones nacionales e internacionales

- Mayor confianza a la hora de emprender fusiones y adquisiciones, debido al mayor nivel de transparencia

- Mejora sustancial de los procesos comerciales, incluyendo controles internos y la supervisión del proceso de toma de decisiones.

\section{Criterios de buenas prácticas de gobierno corporativo}

La necesidad de la existencia de un gobierno, en general, se relaciona con el ejercicio de la autoridad en la toma de decisiones que afectan a los intereses de distintas partes interesadas en los resultados o consecuencias de dichas decisiones. Aunque esto es claro para las estructuras sociales, no lo es tanto para las corporativas. ¿Por qué se haría necesario establecer procedimientos de toma de decisiones al interior de una empresa cuando la competencia de mercado es capaz de asignar eficientemente los recursos en la economía? La respuesta es muy simple: porque en realidad no lo es.

La revisión de la literatura académica sobre el tema, realizada en el marco de esta investigación nos permitió establecer una serie de criterios que no solo aseguran la existencia de un Buen Gobierno Corporativo (BGC), estructurado y eficaz, sino que además explican la necesidad de su existencia en la Empresa moderna. 


\section{Distribución equitativa de las rentas de capital}

Hart (1995) plantea que la mano invisible de la que hablaba Adam Smith en sus escritos es incapaz de asignar eficientemente los recursos en una empresa sin el uso de alguna forma de autoridad debido a la existencia de muchas transacciones económicas "no estandarizadas", principalmente porque que en estas transacciones se generan cuasi-rentas ${ }^{2}$ que no pueden ser asignados en una forma obvia previamente. Las cuasi-rentas se producen debido a la existencia de complementariedad y externalidades en los diferentes aportes de quienes participan en la relación económica que hacen que el valor del todo (la empresa) sea distinto (generalmente mayor) a la suma de sus partes. Las cuasi-rentas no pueden ser repartidas previamente debido a que resulta imposible elaborar contratos perfectos que contemplen la totalidad de las eventualidades que puede sufrir la relación económica establecida, o a que el cumplimiento de dichos contratos no puede ser garantizado debido, por ejemplo, a un sistema judicial deficiente. En otras palabras, cuando la relación económica o empresa genera rentas cuya distribución no puede predeterminarse, el derecho a tomar decisiones posteriores sobre ellas resulta valioso puesto que aumenta el poder negociador para capturarlas. A este derecho se le conoce como el control residual y en las corporaciones modernas recae, en teoría, sobre los accionistas.

Sin embargo, si trascendemos la definición de empresa desde la simple colección de activos físicos bajo un contrato de propiedad común (Grossman \& Hart, 1986) a la de un conjunto de inversiones más o menos específicas en capital físico, capital humano, trabajo, relaciones con proveedores, preferencias de los clientes etc., que no pueden ser replicadas en forma obvia por el mercado (Rajan \& Zingales, 1997), dejaríamos entonces de verla como una propiedad compartida de muchos dueños en la que ninguno tiene suficientes incentivos para invertir recursos en administrar o monitorear la administración de sus activos y que por tanto debe manejarse a través de representantes de los dueños (agentes), en presencia de información imperfecta o al menos asimétrica, lo que genera el tradicional problema de agencia entre los accionistas y los ejecutivos de una empresa, para empezar a visionarla como una relación en la que todos contribuyen y no únicamente los accionistas. A estos contribuyentes se les conoce como stakeholders, y en principio todos ellos desean obtener un retorno razonable por sus inversiones. Es en este escenario cuando cobra importancia el gobierno corporativo, la cual radica en ofrecer un conjunto de condiciones que garanticen a los diferentes stakeholders que podrán non solamente recuperar su inversión, sino además obtener de ella algún dividendo. Esta visión trascendente cuestiona, por lo tanto, la lógica de que todo el derecho al control residual de la corporación recae sobre los accionistas o dueños.

\section{Asignación eficiente de recursos}

No obstante, la importancia del BGC no se limita a la distribución de rentas, sino que tiene, además, un efecto importante sobre la asignación eficiente de recursos (Short, 1994). Por ejemplo, debe evitar, o al menos dificultar, que los participantes en la empresa puedan decidir o bien invertir una cantidad sub-óptima de recursos (un empleado que de manera

\footnotetext{
2.Se denomina cuasi-renta al valor de los ingresos suplementarios que recibe un factor productivo cuando la oferta del mismo no puede aumentarse durante un período determinado, casi siempre relativamente corto. Se produce cuando existen equipos productivos especiales, derivados de innovaciones tecnológicas aún no difundidas, materias primas de difícil acceso en un mercado o habilidades particulares que no poseen los demás hombres y no pueden desarrollarse con facilidad en el corto plazo.
} 
deliberada disminuya su tiempo o cantidad de trabajo, si considera que esa inversión no está siendo adecuadamente recompensada) o por el contrario, invertir más de la cuenta en actividades que aumenten su capacidad negociadora posterior (por ejemplo, un gerente que especializa la empresa en un rubro de su particular experticia o que acapara información, con el fin de hacerse relativamente indispensable).

\section{Retención de los derechos de propiedad por parte de los accionistas}

Desde esta perspectiva, parece claro que todos los diferentes stakeholders de una corporación tienen la misma importancia. Dicho de otro modo, en lo que se refiere a la asignación eficiente de recursos, el gobierno corporativo debería garantizar la imposibilidad o extrema dificultad de expropiación de sus inversiones en la empresa a todos ellos por igual. En la práctica, sin embargo, la corporación moderna parece privilegiar a una clase particular de inversionistas: los accionistas o dueños, quienes retienen para sí el control residual sobre los activos de la empresa y no necesitamos pensarlo mucho para aceptar que así debe ser. Pero, ¿Y si lo pensamos mejor? ¿De dónde procede ese "derecho" que aceptamos tan fácilmente? Probablemente se requiera más de un argumento para respaldarlo.

Primero que todo, los demás stakeholders de la empresa tienen una mayor capacidad de negociación sobre las cuasi-rentas generadas, puesto que el activo productivo con el que contribuyen a la corporación permanece en su poder (Alchian \& Woodward, 1988). Por ejemplo, el trabajador cuyo capital aportado a la empresa es su capacidad de trabajo (física o intelectual), puede decidir en cualquier momento renunciar y llevarse ese capital consigo, valorizado además por la experiencia adquirida durante su estadía en la compañía.

En segundo lugar, si los propietarios de activos en relaciones económicas bajo contratos incompletos o imperfectos son también quienes toman la decisión acerca de cuanto especializarlos, lo harían de manera sub-óptima para evitar ser expropiados en el futuro por agentes con mayor conocimiento e información. Con el fin de lograr un uso eficiente de estos recursos, los proveedores de fondos deben entregar sus activos a la empresa y no participar directamente en la decisión de cuánto especializarlos, reteniendo el control residual en compensación por el hecho de que su capital va a ser especializado para el beneficio de la firma y como garantía de que no les será expropiado (Rajan \& Zingales, 1997).

\section{Alineación de los intereses de los accionistas con los de la empresa}

Los argumentos anteriores implican no sólo que el derecho residual de control de los activos de la empresa debe recaer sobre los accionistas, sino que además, es importante que éstos deleguen en otros agentes las decisiones acerca de cuánto especializarlos y sean capaces de transar sus derechos sobre la empresa en un mercado lo más competitivo posible. Este aspecto último resulta primordial para lograr la alineación de los intereses del accionista con los de la empresa como un todo, a la hora de ponderar la política de inversiones. Esta alineación de intereses sólo es posible cuando el precio de mercado de las acciones de la empresa refleja efectivamente el valor presente de las oportunidades de inversión futuras de los accionistas. Es decir, en un mercado de capitales desarrollado y competitivo. 


\section{Protección de los accionistas contra la expropiación y los costos de agencia}

Si seguimos la lógica de la discusión planteada hasta aquí, según la cual resulta óptimo que los accionistas deleguen la decisión de especialización de las inversiones en otros agentes y que, además, diversifiquen el riesgo asumido mediante un portafolio construido para ese fin, la estructura óptima para una empresa que opera en un mercado de capitales competitivo consistiría en una gran cantidad de accionistas pequeños, propietarios de acciones comunes, que no juegan otro rol en la empresa y que delegan su autoridad en una junta directiva o consejo de administración y un grupo de ejecutivos.

Esta estructura de empresa es considerada un paradigma tradicional desde que Berle y Means (1936) la describieran por primera vez en 1932 en su obra clásica The Modern Corporation and Private Property (a la que Weindenbaun llamó: "uno de esos clásicos perdurables que muchos citan pero pocos han leído", en el prólogo de la edición de 19913).y en ella los accionistas se encuentran expuestos a ser expropiados por sus agentes o a pagar el llamado "costo de agencia"4.

El BGC tiene la capacidad de proteger a los accionistas de la expropiación o costo de agencia a través de tres mecanismos básicos.

En primer lugar, dota a los inversionistas de protección normativa en forma de políticas, directivas y procesos que protegen los derechos de los accionistas y obligan a los ejecutivos a informar veraz y oportunamente al mercado de los resultados operativos y financieros y la toma de decisiones y sus consecuencias. No sobra aclarar que este mecanismo puede resultar innecesario si ya existe una legislación gubernamental en el país en este sentido. En segundo lugar, los accionistas pueden ser protegidos mediante el desarrollo de un sistema de monitoreo, evaluación y control, sobre la gestión y el logro de los objetivos corporativos, el cual advierte periódicamente a los directivos que en caso de descuidar sus deberes y disminuir o poner en riesgo el valor de la empresa, podrían ser reemplazados por personas más dispuestas a alinear sus intereses con los de la compañía.

En tercer lugar, los accionistas pueden protegerse implementando de forma sistemática y debidamente reglamentada incentivos a sus agentes en forma de bonos, remuneraciones variables según logros de altos desempeños, stock options o despidos condicionados.

\section{La recomendaciones de la OECD}

En mayo de 1999 la Organización para la Cooperación y el Desarrollo Económico (OECD, por sus siglas en inglés -Organization for Economics Co-operation and Development) aprobó y publicó por primera vez los "Principios de la OECD para el Gobierno de las Sociedades.", los cuales fueron revisados y ajustados en el 2004 y publicados bajo el título de "Principios de

\footnotetext{
3. La cita original de Weindenbaun es la que sigue: "This monumental work on the corporation is one of those enduring classics that many cite but few have read."

4. Los costos de agencia o problemas de agencia son conflictos que surgen entres los accionistas (principales) y los directivos (agentes) de una empresa, generalmente facilitados por las asimetrías informativas que permiten a los agentes, que buscan la maximización de su propio beneficio, perseguir objetivos distintos a los acordados, para los cuales fueron contratados, disminuyendo o no maximizando las utilidades de los accionistas. También se llama así a los dividendos que los accionistas comparten con los directivos para mantener en ellos el interés por maximizar las utilidades de los primeros.
} 
Gobierno Corporativo de la OECD" (2004). Estos principios resumen una serie de iniciativas y experiencias de países miembros de esa organización y en particular, constituyen una serie de recomendaciones en las áreas de: derechos de los accionistas, tratamiento equitativo a los accionistas, función de los stakeholders en el gobierno corporativo, y rol del directorio. No podemos pasar por alto, sin embargo, que la misma OECD reconoce que no existe un modelo único de gobierno corporativo, o uno de superior desempeño sobre los demás, ya que su eficacia depende de muchos factores, entre ellos, la normatividad vigente en el país de operación de la empresa, la concentración de capital, la estructura de propiedad, etc. A pesar de ello, sí establece que existe un conjunto de elementos comunes a todo BGC y trata de recopilar en sus principios los que considera, dada la experiencia de los países miembros, los más importantes.

Algunos de ellos, como el reconocimiento del papel privilegiado de deben jugar los accionistas frente a los restantes stakeholders y la garantía y protección de su derecho a mantener la propiedad residual de los activos de la empresa, ya fueron abordados antes en este escrito, por lo tanto nos concentraremos en los restantes.

En estrecha relación con el ítem anterior, se establece que los accionistas deben ostentar derechos iguales entre ellos y que debe promoverse toda forma de proporcionarles información oportuna, suficiente y adecuada respecto a las decisiones que los ejecutivos tomen y que resulte crítica para el buen desempeño de la empresa. Se recomienda también que la adquisición del control corporativo esté debida y completamente reglamentada de forma que sea transparente y cuando se realice, se haga en condiciones justas y equitativas para todos los participantes. También, se recomienda establecer mecanismos regulatorios que aseguren que la corporación y sus controladores respetarán los derechos e intereses de los restantes stakeholders de la compañía, entregándoles en forma oportuna y adecuada la información relevante. Finalmente, se establecen las principales responsabilidades y derechos de los miembros del directorio de la corporación. En este sentido, los directores deben actuar en forma diligente, asegurándose que las decisiones que se tomen afecten a los diferentes accionistas por igual. Además, deben ser capaces de tener una opinión informada e independiente de la que sostengan los ejecutivos de la empresa.

Las recomendaciones de la OECD para el BGC no han estado exentas de polémica (Santonja \& Borges, 2005). Sobre todo se les ha hecho de forma reiterada dos críticas: por un lado, la rigidez de la estructura de gobierno corporativo que surge de estas recomendaciones, contradice lo que la propia OECD sostiene, acerca de la posibilidad de que existan diversos tipos de gobierno corporativo aplicables en diferentes contextos económicos e institucionales. Y por el otro lado, se critica la excesiva importancia que le dan las recomendaciones al accionista, incluso en desmedro de otros stakeholders, que parecieran no considerarse importantes.

\section{ESTUDIO DE CASOS EMPRESARIALES}

Tanto Coosalud ESS EPS-S como Mutual SER ESS EPS-S son Empresas Solidarias de Salud pertenecientes al sector de economía solidaria creadas a principios de los años 90 por miembros de las comunidades pobres y vulnerables de Cartagena de Indias y El Carmen de Bolívar, respectivamente, en el marco del programa de Empresas Solidarias de Salud, instituciones creadas por iniciativa del Ministerio de Salud y el Departamento Nacional de Planeación (DNP) avalado por el Consejo Nacional de Política Económica y Social (CONPES) (1993). 
Coosalud fue creada el 20 de junio de 1994 por iniciativa de 800 líderes cívicos de la zona Suroriental de Cartagena, quienes aprobaron sus estatutos y su estructura organizativa, con un patrimonio inicial de apenas un millón de pesos (alrededor de US \$500), siendo la primera empresa solidaria de salud de Cartagena.

Mutual SER, por su parte nació bajo el nombre de Mutual Montes de María, como uno de los proyectos que planteó la Asociación de Municipios del mismo nombre. La posibilidad de administrar recursos y servicios de salud para sus propias comunidades fue considerada por la Asociación de Municipios que en 1993, con la asesoría del PNR y la Corporación por la Paz y el Desarrollo Comunitario, Corpadec, se presentó un proyecto de constitución de Empresa Solidaria de Salud ante el Ministerio de Salud. El proyecto se aprobó y de esta manera se constituyó Mutual Montes de María. Se destaca en la primera etapa de constitución de la empresa, la selección de los socios realizada por los Consejos Municipales de Rehabilitación, CMR, formados dentro del PNR. Hacían parte de estos consejos la comunidad y las autoridades formales de estos municipios, lo cual garantizaba que el proceso fuera democrático y legitimado. Se logró en esta etapa la asociación de 55.000 personas, representadas por 7.800 cabezas de familia, en un término aproximado de dos meses, sin la utilización de instrumentos técnicos de focalización, pero sí por decisión de la propia comunidad que identificaba a las personas más vulnerables y pobres que debían ser asociadas (Mejía Reyes et al., 1999). Posteriormente se fusiona con la Asociación Mutual de Bolivar -Amuvar, para transformarse en la Asociación Mutual SER.

A pesar de sus inicios modestos, ambas empresas se consolidaron rápidamente como líderes del sector de aseguramiento en salud de la región caribe aprovechando las oportunidades que la Ley 100 de 1993 le brindaba a estas organizaciones comunitarias. Hoy día son dos de las más grandes Empresas Promotoras de Salud del Régimen Subsidiado (EPS-S) del país, contando Coosalud con más de 1'600,000 afiliados repartidos en 15 departamentos y Mutual SER con cerca de 1'200,000 y presencia en todos los departamentos de la región caribe colombiana.

Las dos organizaciones tienen en común además de su origen comunitario y su naturaleza solidaria que su desarrollo corporativo fue liderado por personas que aún hoy se encuentran en la alta dirección de la Empresa y esa fue una de las razones por las que decidimos utilizarlas como objeto de estudio. Sus dos líderes actuales, Jaime González Montaño (Coosalud) y Galo Viana Muñoz (Mutual SER) lo han sido durante los últimos quince años por lo que se encuentran en una posición inmejorable para hacer un recorrido en el tiempo sobre la importancia crítica del BGC en el logro del éxito empresarial que ambas instituciones ostentan.

Con cada uno se adelantó una entrevista estructurada de casi dos horas de duración, utilizando las preguntas como excusa para descubrir en conjunto la influencia que la gobernanza ha ejercido en la institucionalidad de la compañía y les ha permitido no solo sobrevivir en un sector turbulento y de constante cambio, como lo es el del aseguramiento en salud en Colombia, sino además destacarse como dos de las mejores EPS-S del país.

\section{Coosalud ESS EPS-S}

Para González Motaño, el BGC de Coosalud ha mostrado evidencias palpables de haber contribuido al incremento del valor de la empresa, en la atracción y retención de recursos de capital y en la disminución del costo de los mismos. Aunque por tratarse de una 
cooperativa, no se puede realizar con ella el mismo ejercicio que con una sociedad por acciones, el interés real de inversionistas nacionales y extranjeros de poner al servicio de la gestión de la compañía importantes capitales líquidos para el desarrollo de proyectos estratégicos, por ejemplo, son una clara demostración que la valoración de la empresa ha aumentado considerablemente en los últimos diez años. Particularmente, en dos ocasiones cuando se buscó financiamiento para el desarrollo de grandes proyectos de desarrollo institucional se consiguió en la primera de ellas contar con un capital de alrededor de 50 mil millones de pesos (unos US \$25 millones) por parte de inversionistas individuales y en la segundo el visto bueno de dos fondos de inversión, uno colombo-americano y otro español, este último valoró específicamente los avances de la empresa en responsabilidad social empresarial. En las dos ocasiones, los costos de capital fueron competitivos. Sin embargo, reconoce que debido a la inestabilidad del sector salud el costo de capital en el sector financiero, sobre todo el bancario, no ha sido influido por el BGC de la Cooperativa.

También resulta evidente la influencia positiva del BGC en la capacidad de la Empresa para enfrentar y responder a las presiones externas del mercado. Este aspecto se hizo palpable hace tres años cuando Coosalud fue sujeta de una vigilancia especial por parte de la Superintendencia Nacional de Salud que puso en interdicción su continuidad como aseguradora de salud. En opinión de González, fue la confianza generada por la estructura de gobierno corporativo en los principales proveedores de servicios de la Empresa, lo que le permitió salir avante en esta crítica situación, ya que sin ninguna garantía diferente a las promesas de cumplimiento de los compromisos adquiridos este grupo de proveedores continuó prestando los servicios que se requerían para el normal funcionamiento de la EPS-S, apalancando la operación de la misma en el periodo de crisis y permitiéndole superar la intervención incluso antes del periodo previsto, con muy poca afectación de su capacidad operativa.

El BGC desarrollado en Coosalud hay sido piedra angular en el proceso de equilibrar los intereses divergentes de las cabezas visibles de la institucionalidad solidaria (delegados y consejeros) y una herramienta de invaluable utilidad para resolver los problemas generados por dichos intereses. El crecimiento y la maduración corporativa de estas personas, producto del entendimiento y aceptación de los principios de BGC, han llevado a que cada año los intereses personales se hallan alineado cada vez más con los corporativos y al entendimiento de que la institución y más importante y trascendente que cada uno de sus miembros. Como resultado, hoy Coosalud cuenta con órganos de dirección y de vigilancia y control convencidos y comprometidos con los objetivos estratégicos de la organización, permitiéndole a la alta dirección concentrarse en la creación de valor para sus stakeholders. González Montaño está completamente seguro que el BGC es un pilar fundamental que garantiza la sostenibilidad de la Empresa, además que en la actualidad su estructuración y fortalecimiento es sujeto de vigilancia por parte de la Superintendencia Nacional de Salud, por ser un requisito normativo para la habilitación de la empresa como aseguradora de salud.

También considera el Gerente General de Coosalud que el BGC ha constituido un factor importante para atraer y retener altos directivos que garanticen el éxito en la implementación de estrategias corporativas y ofrece como prueba de ello la baja rotación de personal en ese nivel y el reclutamiento para estos cargos de profesionales de alta valoración en el mercado laboral.

Así mismo, califica con 5 sobre 5 la influencia del BGC en la consecución de mejores resultados operativos. Siendo una Empresa que adelanta un proyecto para ser considerada 
uno de los mejores lugares para trabajar del país, se ha puesto especial énfasis en la transparencia, la claridad en el direccionamiento, el control, la provisión adecuada de recursos, la formación continua, la retroalimentación y la evaluación constante del logro de objetivos como mecanismos que generan un ambiente laboral agradable sin menoscabo de la productividad y la competitividad.

La revisión de la historia corporativa de Coosalud demuestra que la estructuración del gobierno corporativo no ha sido el fruto de la ejecución de un plan formalizado y esta es quizá la principal razón por la que algunas medidas importantes no han sido tomadas de manera premeditada. Ásí, por ejemplo, los Códigos de Buen Gobierno y de Ética fueron inicialmente elaborados en respuesta a la exigencia normativa y solo unos años después se revisaron y ajustaron de manera más sistemática. Además, debido a la naturaleza democrática de la elección de los miembros del Consejo de Administración, así como a la imposibilidad normativa de incluir en dicho órgano agentes externos a la Cooperativa, la empresa no puede influir en asegurar su composición apropiada para el ejercicio del BGC, en términos de tamaño, experiencia y combinación de habilidades, garantizar la independencia de sus miembros o establecer la existencia y operación de comités especializados a su interior que garantice el análisis en profundidad de los aspectos más relevantes de la operación, lo cual la mayoría de las veces no se da.

A pesar de estas importantes limitantes, si se han hecho en el pasado reciente esfuerzos importantes para mejorar el ejercicio de dirección del Consejo de Administración, con lo que se ha logrado elaborar e implementar procesos y procedimientos de dirección y mantener relaciones de independencia entre sus miembros y la Alta Gerencia de la Organización.

Por otra parte, establecer la existencia y operación de comités especializados dentro del Consejo de Administración que garantice el análisis en profundidad de los aspectos más relevantes de la operación, realizar evaluaciones del desempeño de sus miembros, contar con mecanismos que permitan prestar atención a varios detalles para lograr efectividad en sus funciones, contar con un sistema de monitoreo para medir el desempeño y la evaluación y remuneración de la Gerencia y contar con un plan de sucesión de los altos directivos son retos que aún no se han abordado adecuadamente pero que están en la agenda de los desarrollos futuros.

González Montaño destaca que el BGC ha mostrado su mayor eficacia en la superación de los conflictos de agencia, que se vuelven particularmente relevantes en el caso de la Empresa Solidaria de Salud ya que su composición de propiedad se asemeja muchísimo a la empresa paradigmática de Berle y Means (1936), es decir, un número muy grande de pequeños accionistas (Coosalud cuenta con más de 11.000 asociados, todos con los mismos derechos y deberes) que delegan su autoridad en un Consejo de Administración y una estructura administrativa, con el agravante de que la normatividad vigente en Colombia les impide asegurar la adecuada composición de este Consejo de Administración y prácticamente no tener influencia o control a través de él sobre la Alta Gerencia de la Empresa. En este panorama, sólo el BGC pareciera tener la capacidad para generar la suficiente confianza en los asociados de que sus intereses estarán bien cuidados en manos de la administración, confianza que ha sido honrada, a la luz de los resultados: Coosalud pasó de ser una Empresa local de Cartagena con 50.000 afiliados y radio de acción en el norte del departamento de Bolívar a una Empresa del ámbito nacional con más de 1,6 millones de afiliados en 15 departamentos.

A partir del 2004, Coosalud empezó a trabajar de manera consciente y sistemática en el fortalecimiento del BGC, siendo la identificación de los potenciales promotores una de 
las primeras actividades adelantadas. Sin embargo, los restantes pasos identificables en la correcta estructuración de un BGC, como la identificación de sus promotores, el desarrollo de un entendimiento común de arriba hacia abajo, el entendimiento de las fuentes de resistencia y la adopción de medidas para superarlas y la modificación de actitudes de los asociados, gerentes, directores y trabajadores en pro de la implementación del BGC, no se dieron de forma sistemática y más bien fueron producto de intenciones mal definidas por fortalecer la institucionalidad de la Cooperativa. En este aspecto Viana Muñoz llama la atención sobre la generación en la empresa, en todos los niveles, de un ambiente que permite la generación de resistencias, las cuales son percibidas más como oportunidades de mejora y de identificación de fallas y errores, aunque no se hace de manera sistemática y planificada.

Aunque se han fijado prioridades y se han establecido fuentes de asesoramiento en el desarrollo y fortalecimiento del BGC, apenas se está construyendo una hoja de ruta para su fortalecimiento y no se han realizado evaluaciones comparativas de su estructura, función o resultados.

En el concepto del Gerente General, Coosalud ha experimentado un retroceso en el aspecto de formalización de las políticas y directrices que lleven a su implementación operativa. Este aspecto, que fue muy fuerte en el pasado reciente, ha sido desatendido en los últimos años, pero hoy día se plantea la necesidad de retomarlo. En este mismo sentido, si bien la normatividad del sector solidario impide la presencia de externos en el Consejo de Administración, en Coosalud esta limitante se ha enfrentado con un fuerte componente de capacitación, que empezó por la profesionalización de los delegados (hoy día todos los miembros del Consejo de Administración, el Comité de Educación y la Junta de Vigilancia son profesionales universitarios, formación que recibieron apoyados económicamente por la Cooperativa) y su especialización en temas de planeación, desarrollo organizacional y administración, de tal manera que hoy este órgano de dirección ha visto aumentado exponencialmente su capacidad de dirigir y controlar los destinos de la organización. Como ya se comentó antes, es imposible garantizar, dado el marco legal, la composición apropiada del Consejo de Administración y la independencia de sus miembros, que al ser elegidos democráticamente necesariamente responden a los intereses de sus electores.

En lo que sí es fuerte la empresa es en la garantía de los derechos de los asociados y la transparencia. La asamblea general de delegados, por ejemplo, es una tradición a la que se le da mucha importancia. Por la naturaleza misma del estamento cooperativo cada miembro de la Asamblea de Delegados tiene el mismo derecho a voz y voto que los restantes y sus representaciones en el Consejo de Administración son igualitarias. De igual forma los mecanismos de rendición de cuentas a que obliga la Ley a las EPS-S son asumidos en Coosalud con un alto compromiso, con lo que se cumple a cabalidad con la comunicación a los diferentes grupos de interés el resultado del desempeño económico, social y ambiental de la operación de la Empresa. En cuanto a la organización y divulgación de la información crítica para la toma de decisiones claves y sus resultados y el establecimiento de mecanismos para mejorar el entorno de control, González Montaño considera que faltan desarrollos importantes.

Finalmente, para el Gerente General de Coosalud son más que evidentes los beneficios que el BGC le ha traído a la Empresa, sobre todo en mejorar la reputación de la empresa, lo cual es más que evidente dado la confianza manifiesta de afiliados y proveedores y del clima organizacional, lo cual se ha podido objetivar por sucesivas mediciones realizadas por Hay Group, Great Place to Work y Human Capital. En cambio no considera que el BGC haya 
tenido mayor influencia en el aumento de los ingresos operacionales y no operacionales o la disminución de los costos de operación, más allá de contribuir al fortalecimiento de la confianza en los proveedores.

\section{Mutual SER ESS EPS-S}

Galo Viana Muñoz, médico de profesión, Gerente y líder organizacional de Mutual SER desde hace más de 10 años, considera que las prácticas propias del BGC en esta empresa han estado fuertemente ligadas a su origen comunitario y naturaleza solidaria. Resalta como beneficios evidentes la mejora de la imagen como institución, separándola de la de sus conductores. Si bien considera que el BGC ha realizado una contribución modesta al crecimiento extraordinario de la empresa, piensa que sí es destacable su influencia en el fortalecimiento de la institucionalidad de la misma.

El énfasis que Mutual le ha dado a la estructuración del BGC se hace más evidente en la manera como actualmente se maneja el poder dentro de la organización, lo que ha permitido la reglamentación de los patrones de decisión de muchos aspectos importantes del funcionamiento de la Empresa, como la contratación de bienes y servicios y la vinculación de personal. El BGC y la credibilidad que su fortalecimiento ha generado tanto en asociados como en trabajadores, ha sido un factor crítico para equilibrar los intereses de los miembros de la Junta Directiva y la Alta Gerencia con los de la empresa.

Desde hace varios años Mutual SER realiza encuestas de percepción de la Empresa entre afiliados y proveedores a través del Centro Nacional de Consultoría, las cuales han evidenciado la mejora sostenible de su imagen ética y de transparencia, así como un aumento importante en la confianza de los proveedores. Los resultados de estas encuestas son tomados como criterio de evaluación del desempeño de los altos directivos, lo cual demuestra que el grado de exigencia de dichas evaluaciones es relativamente alto. Además, existe en la Empresa un proceso continuo de evaluación de la calidad de la atención al afiliado por parte de los miembros de los órganos de dirección, lo que lo aumenta aún más. Por otro lado, se establecen metas a las que se les hace seguimiento y se premia su cumplimiento.

Viana le da un alto valor al BGC como vehículo para la formalización de los procesos al interior de la organización y la credibilidad en la transparencia de su aplicación de la que los ha dotado. Al mismo tiempo considera que esta formalización de los procesos ha fortalecido el BGC generando un círculo virtuoso que le ha permitido a la empresa avanzar en varios aspectos de desarrollo institucional. En su opinión, los ejemplos constantes de aceptación de las decisiones gerenciales en asuntos tan delicados como el reclutamiento, promoción y despido de personal, la selección y contratación de proveedores y el pago de servicios prestados, son el mejor barómetro del impacto que el BGC ha tenido en el desarrollo institucional. Hoy podemos decir -afirma- que las personas vinculadas con la Empresa saben, y actúan en consecuencia, que los procesos son claros y transparentes, y que son escasas las situaciones en que se espera o se sospecha la intervención de personas con intereses propios para inclinar decisiones en un sentido u otro.

El Gerente General de Mutual SER está convencido que el aumento del valor de la Empresa (calculado en el número de afiliados), la mejora en la sostenibilidad de la misma, que hoy se presenta más estable y equilibrada que nunca antes, su alta capacidad para atraer y retener talento humano valioso, la mejora continua de los resultados operativos y la capacidad manifiesta de la organización para responder a las exigencias del mercado, son en buena 
parte consecuencia de los avances logrados a través del desarrollo del BGC. En cambio, considera que su influencia ha sido poca o nula en los aspectos relacionados con el acceso a capital y su costo.

En Mutual SER la estructuración del BGC no ha sido producto de una planeación deliberada, sino más bien del deseo de la Junta Directiva y la Alta Gerencia de dotar a la persona jurídica de una mayor autonomía y capacidad institucional. Esta es quizá la razón por la que no se han fijado prioridades en este sentido, no se han realizado evaluaciones comparativas, no se han establecido fuentes de asesoramiento ni se ha diseñado una hoja de ruta. Sin embargo, esto no ha sido óbice para que se hayan surtido importantes pasos en la estructuración y fortalecimiento del BGC, tales como: la superación de los conflictos de agencia aunando los intereses de asociados y gerentes, lo que principalmente se ha logrado a través de la formalización de procesos y procedimientos en constante perfeccionamiento; el desarrollo de un entendimiento común de arriba hacia abajo, evidenciado en el énfasis que se ha puesto siempre a la educación y capacitación de los asociados y miembros de los órganos de control de Mutual, que permite que hoy en su totalidad sean profesionales universitarios; entender las fuentes de resistencia y adoptar medidas para superarlas y modificar las actitudes de asociados, gerentes, directores y trabajadores en pro de la implementación del BGC.

Mutual SER cuenta con un Código de Buen Gobierno y un Código de Ética, los cuales han sido desarrollados para su implementación a través de políticas, reglamentos y procesos. El carácter democrático de la elección de los miembros de la Junta Directiva y otros órganos de dirección y control de Mutual, obligado por la normatividad vigente en el país para las empresas de economía solidaria, impiden asegurar su composición de manera apropiada para el ejercicio del BGC en términos de tamaño, experiencia y combinación de habilidades y la independencia de sus miembros.

Al interior de la Junta Directiva existen diferentes comités especializados que garantizan el análisis en profundidad de los aspectos más relevantes de la operación, pero no se realizan evaluaciones del desempeño de sus miembros.

Resulta especialmente destacable la existencia de un plan de sucesión de largo plazo representado en el Programa SER Joven, en el cual se han identificado más de 70 jóvenes relacionados con la empresa a través de asociados, trabajadores o directivos, con los cuales se están llevando a cabo procesos de sensibilización, educación, formación y capacitación que los lleven a convertirse en los líderes del futuro de la organización.

Viana Muñoz asegura que en Mutual SER han sido especialmente celosos con la protección de los derechos de los asociados, aspecto que forma parte del ADN de la Empresa por causa de su origen comunitario y mutualista. Es especialmente destacable el proceso de rendición de cuentas, el cual se realiza en todos los sitios donde opera la empresa, con un rigor que ya se ha vuelto tradicional y que incluye desde los aspectos financieros y administrativos hasta los operacionales y de prestación de servicios, pero que además se enriquece con actividades de capacitación que mejoran la capacidad de convocatoria y animan a la participación activa de los asistentes. Es mediante este proceso que se asegura la comunicación del resultado del desempeño económico, social y ambiental de la operación de la Empresa a los diferentes grupos de interés de la misma. En este mismo sentido se han alcanzado desarrollos importantes en el sistema de información de la empresa que permite a la alta dirección acceder a información crítica para la evaluación de resultados y la toma de decisiones. 
Para el Gerente General de Mutual SER, el BGC ha influido positivamente en la mejora del clima organizacional y la disminución de la rotación del personal, pero principalmente en la mejora de la reputación de la Empresa. En su concepto, el BGC le ha permitido a Mutual aumentar el grado de compromiso de sus trabajadores, que al sentirse objetivamente evaluados en su desempeño le agregan valor al cumplimiento de sus obligaciones laborales. La confianza en la transparencia y justicia del proceso de evaluación hacen aún más valiosos los premios por el cumplimiento de los objetivos fijados (que se reconocen públicamente en un evento del tipo "la noche de los mejores"). Esta irradiación de confianza y transparencia, que el BGC hace a toda la organización, y que genera compromiso por el buen desempeño, es quizá lo que le ha permitido a Mutual SER encabezar los más recientes rankings de EPS-S a nivel nacional, fundamentalmente por sus altas calificaciones en los aspectos relacionados con la calidad del servicio prestado, y que en diversas ocasiones el Ministro de Salud y la Protección Social haya expresado públicamente que la considera una de las mejores EPS-S del país.

\section{CONCLUSIONES}

El Gobierno Corporativo se ha convertido en uno de los temas empresariales y de responsabilidad social más seriamente debatidos en la actualidad, centrando el debate en su importancia en el marco de la Economía de Mercado, tanto para impulsar mejores resultados económicos como para proteger los derechos de los inversores. En nuestra revisión bibliográfica se hizo evidente la mayor consciencia por parte de inversores, reguladores y directivos de empresas acerca del aporte que la práctica de un Buen Gobierno Corporativo (BGC) puede suponer en términos de una mayor eficiencia en la colocación de capitales y a un más alto nivel de confianza en los mercados financieros. También se evidenciaron los beneficios que pueden alcanzar las organizaciones a través del BGC, entre los que se destacan: acceso a mercados de capital y disminución de su costo, aumento del valor de mercado y la rentabilidad de la empresa, mejora en los resultados operativos (aumento de los ingresos y disminución de los costos de operación) y mejora en la reputación de la empresa.

Las recomendaciones para la implementación de un BGC encontradas en la literatura revisada están basadas en su mayoría en estudios y análisis en profundidad de experiencias exitosas tanto a nivel empresarial como de gremios y sectores de la economía y aunque no hay soluciones simples ni un modelo único que se adapte a todos los casos, si es posible establecer una serie de variables que mejoran la posibilidad de una implementación exitosa de BGC, entre ellas: la distribución equitativa de las rentas de capital, la asignación eficiente de recursos, la retención de los derechos de propiedad por parte de los accionistas, la alineación de los intereses de los accionistas con los de la empresa, la protección de los accionistas contra la expropiación y los costos de agencia, la igualdad de derechos entre los accionistas, la garantía de acceso por parte de los accionistas y otros stakeholders a información oportuna, suficiente y adecuada respecto a las decisiones de los ejecutivos, la debida reglamentación de la adquisición del control corporativo.

El estudio de los casos de Coosalud ESS EPS-S y Mutual SER ESS EPS-S, dos empresas promotoras de salud del régimen subsidiado, realizada a través de entrevistas estructuradas a cada uno de sus respectivos Gerentes Generales, demostró que el BGC fue identificado como un impulsor importante del desarrollo y crecimiento empresarial y como principal factor contribuyente para el alcance de importantes beneficios corporativos. 
En Coosalud se identificaron como beneficios del BGC el incremento en el valor de la empresa, captación y retención de inversionistas y diversificación del capital de trabajo (en forma de patrocinadores de proyectos), la capacidad de la empresa para enfrentar y responder a las presiones del mercado, equilibrar el interés de los accionistas, resolver problemas generados por intereses propios de los asociados, los órganos de dirección y la alta gerencia, el logro de mejores resultados operativos, mejora en la reputación de la empresa, mejora del clima organizacional y disminución de la rotación del personal.

Por su parte en Mutual SER, se identificaron como beneficios derivados el BGC: el desarrollo de capacidades para enfrentar y responder las presiones del mercado, equilibrar los intereses divergentes de los asociados, resolver problemas generados por los intereses propios de miembros de la Junta Directiva o la Alta Dirección de la Empresa, garantizar la sostenibilidad de la Empresa, atraer y retener altos directivos que garanticen el éxito en la implementación de estrategias corporativas, lograr mejores resultados operativos, aumentar los ingresos operacionales y no operacionales, mejorar la reputación de la empresa, mejorar el clima organizacional y disminuir la rotación del personal.

\section{REFERENCIAS BIBLIOGRÁFICAS}

Aguirre Brand, M. L. (2010). Gobierno corporativo en la sociedad por acciones. Santiago: Thomson Reuters Puntolex.

Alchian, A. A., \& Woodward, S. L. (1988). The Firm is Dead; Long Live the Firm: A Review of Oliver E. Williamson's The Economic Institutions of Capitalism. Journal of Economic Literature, 26(1), 65-79.

Berle, A. A., \& Means, G. G. C. (1936). The modern corporation and private property: Transaction Publishers.

Black, B. S., Jang, H., \& Kim, W. (2006). Does corporate governance predict firms' market values? Evidence from Korea. Journal of Law, Economics, and Organization, 22(2), 366-413.

Cano Morales, A. M. a., Orduz Aguilar, C. I., \& Hoyos Ramírez, A. E. (2007). Gobierno corporativo : principales desarrollos en las empresas que cotizan en la bolsa de valores de Colombia (1. ed.). Medellín Colombia: Sello Editorial, Universidad de Medellín.

Carney, M. (2005). Corporate Governance and Competitive Advantage in Family?Controlled Firms. Entrepreneurship Theory and Practice, 29(3), 249-265.

Coffee Jr, J. C. (2002). Racing towards the top?: The impact of cross-listings and stock market competition on international corporate governance. Columbia law review, 1757-1831.

Da Silveira, A. D. M., \& Barros, L. (2007). Corporate governance quality and firm value in Brazil. Universidade de Sao Paulo, Working Paper.

Flores Konja, J. V., \& Rozas Flores, A. E. (2013). El gobierno corporativo: un enfoque moderno. Quipukamayoc, 15(29), 7-22.

Friedman, M. (1962). Capitalism and freedom. Chicago: University of Chicago Press.

Gompers, P. A., Ishii, J. L., \& Metrick, A. (2001). Corporate governance and equity prices: National bureau of economic research. 
DAVID JERVIS JÁLABE, JULIO OROZCO AFRICANO, LUIS ORTIZ IBAÑEZ, JANNINA ALVIS ZAKZUK,

TULIA BELTRAN VENEGAS

El gobierno corporativo como factor crítico del éxito empresarial en el sector salud

Gorbaneff, Y., Torres, S., \& Contreras, N. (2004). Anatomía de la cadena de prestación de salud en Colombia en el régimen contributivo. Gerencia y Políticas de Salud, 3(6).

Grossman, S. J., \& Hart, O. D. (1986). The costs and benefits of ownership: A theory of vertical and lateral integration. The Journal of Political Economy, 691-719.

Hart, O. (1995). Corporate governance: some theory and implications. The economic journal, 678-689.

Hellwig, M. (1998). On the economics and politics of corporate finance and corporate control. Manheim: Universität Mannheim \& Sonderforschungsbereich 504.

IFC/OECD/GCGF. (2010). Guía Práctica de Gobierno Corporativo. Experiencias del Círculo de Empresas de la Mesa Redonda Latinoamericana (pp. 288). Washington D.C.: Internacional Financial Corporate.

Klapper, L. F., \& Love, I. (2004). Corporate governance, investor protection, and performance in emerging markets. Journal of corporate Finance, 10(5), 703-728.

Klein, M. (2013). Firms Behaving Nicely: Incentives and Commitment. Washington DC: International Finance Corporation.

Leal, R. P., \& Carvalhal da Silva, A. (2005). Corporate governance and value in Brazil (and in Chile). Available at SSRN 726261.

Lemmon, M. L., \& Lins, K. (2003). Ownership Structure, Corporate Governance, and Firm Value: Evidence from the East Asian Financial Crisis. The Journal of Finance, 58(4), 1445-1468.

Mayer, C. P. (2013). Firm commitment : why the corporation is failing us and how to restore trust in it (1st ed.). Oxford: Oxford Univ. Press.

Mejía Reyes, S., Ruiz Gómez , F., Acosta Ramírez, N., Eslava Rincón, J. I., Puente Burgos, C. A., Ardila Carrillo, Z., \& Peñaloza Quintero, E. (1999). Entorno, aseguramiento y acceso en el Régimen Subsidiado en Colombia: seis casos de acceso (pp. 199). Santafé de Bogotá: Pontificia Universidad Javeriana/Fundación Ford/Fundación Corona/Cendex.

Morck, R., Wolfenzon, D., \& Yeung, B. (2004). Corporate governance, economic entrenchment and growth: National Bureau of Economic Research.

OECD. (2004). Principios de gobierno corporativo de la OECD (pp. 68). Paris.

Quintana, J. (2012). Guía práctica para el buen gobierno de las empresas familiares. Instituto de la Empresa.

Rajan, R. G., \& Zingales, L. (1997). Power in a Theory of the Firm: National Bureau of Economic Research.

Restrepo Zea, J. H. (2004). ¿ Qué enseña la reforma colombiana sobre los mercados de salud? Gerencia y Políticas de Salud, 3(6).

Rodríguez, J. C. P.-A. (2004). El gobierno corporativo como estrategia de creación de valor. Indret: Revista para el Análisis del Derecho(1), 182.

Salud, D. U. M. d. (1993). Documento CONPES SOCIAL DNP-UDS-Minsalud 001. (0001). Bogotá D.C.: Departamento Nacional de Planeación.

Santonja, A. O., \& Borges, A. M. (2005). Teoría y práctica del buen gobierno corporativo: Marcial Pons. 
Shleifer, A., \& Vishny, R. W. (1989). Management entrenchment: The case of manager-specific investments. Journal of financial economics, 25(1), 123-139.

Short, H. (1994). Ownership, control, financial structure and the performance of firms. Journal of economic surveys, 8(3), 203-249.

Turrent, B., \& del Carmen, G. (2012). Factores institucionales que inciden en la transparencia del gobierno corporativo: un estudio en empresas cotizadas.

Williamson, O. E. (1988). Corporate finance and corporate governance. The Journal of Finance, 43(3), 567-591.

Wong, S. C. (2008). Developing and implementing corporate governance codes. Private Sector Opinion Issue(10), 10-31. 